\title{
Analyse des zones de cisaillement naturelles
}

\author{
Natural shear zones analysis
}

\author{
C. LAMOUROUX \\ Université des Sciences et Techniques de Lille-Flandres-Artois, \\ Laboratoire de Géologie Physique* \\ P. DEBAT \\ Université Paul-Sabatier, Laboratoire de Géologie Structurale et de Tectonophysique** \\ P. SIRIEYS \\ Institut Dolomieu, Laboratoire de Géologie Alpine * *
}

Rev. Franç. Géotech. n 56 , pp. 57-65 (juillet 1991)

\section{Résumé}

L'analyse de la déformation et du déplacement dans des zones de cisaillement ductiles naturelles, développées dans des roches différentes (granodiorite et calcaire), met en évidence des analogies morphologiques mais surtout, des différences géométriques notamment dans l'allure des courbes de glissement (trapézoïdale ou gaussienne). Cette différence traduit des hétérogénéités de déformation dépendant de la composition polyminérale ou monominérale des roches et du comportement des constituants minéraux.

\footnotetext{
Abstract

The analysis of the deformation and displacement of natural ductile shear zones in different rocks (granodiorite and limestone) reveals morphological analogies and geometrical differences in particular in the trend of the shear curves (trapezoidal or gaussian). This last difference indicates strain heterogeneities depending on the rock composition (polymineral or monomineral) and on the constituting behaviour.
}

- Bat. SN5, 59655 Villeneuve-d'Ascq Cedex.

$\therefore$ 38, rue des Trente-Six-Ponts, 31400 Toulouse.

... 15, rue Maurice-Gignoux, 38031 Grenoble Cedex. 
Les zones de cisaillement sont des zones de faible épaisseur finie par rapport à leur étendue, caractérisées par de très fortes déformations matérialisées par divers types de structures planaires résultant d'un déplacement relatif de deux compartiments euxmêmes indéformés. Elles peuvent présenter différents caractères : des zones fragiles, fragiles-ductiles et ductiles sont habituellement distinguées (RAMSAY, 1980). Les zones ductiles naturelles ont été reconnues depuis longtemps (TEALL, 1885) mais la première étude synthétique avec description des principaux types et analyse mathématique de la déformation et évaluation du déplacement est assez récente (RAMSAY et GRAHAM, 1970). Depuis, de très nombreux travaux ont décrits les zones de cisaillement à toutes les échelles, du cristal au continent (RAMSAY et ALLISON, 1979 ; SIMPSON, 1983 ; vol. 2, 1980 et vol. 7, 1987 du Journal of Structural Geology; LAMOUROUX et al., 1991) les interprétations cinématiques reposant pour l'essentiel sur les travaux de RAMSAY (1980) et de RAMSAY et HUBER (1987). En suivant ces auteurs, nous considérons que les zones de cisaillement résultent d'une déformation par cisaillement hétérogène avec ou sans variation de volume qui peut être caractérisée, ainsi que le déplacement associé, par la géométrie de structures induites telles que la foliation ou de marqueurs préexistants (foliations antérieures, filons,...).

Dans ce travail, après un bref rappel de la cinématique des zones de cisaillement, nous examinons des exemples de telles zones développées naturellement dans deux roches différentes: granodiorite du massif du Néouvielle (Pyrénées-centrales) et calcaire de l'encaissant du massif de la Maladetta (Pic de la Tuca Arnau, Sierra Negra, Pyrénées-centrales). Après quantification de la déformation (et du déplacement) en différents points des zones, les problèmes posés par sa localisation et son hétérogénéité sont discutés.

\section{DÉFORMATION PAR CISAILLEMENT : CARACTÈRES GÉNÉRAUX}

Le cisaillement homogène (ou glissement simple) est une déformation rotationnelle caractérisée, dans le référentiel xyz avec l'axe ox parallèle à la direction de glissement et laxe oz perpendiculaire au plan de glissement $x y$, par le champ de déplacement (indépendant de $x$ et $y$ ) :

$$
\begin{aligned}
& \mathrm{u}=\gamma \mathrm{z} \\
& \mathrm{v}=\mathrm{w}=0
\end{aligned}
$$

$\mathrm{u}, \mathrm{v}$ et $\mathrm{w}$ sont les composantes du déplacement selon $\mathrm{x}, \mathrm{y}$ et $\mathrm{z}$ et $\gamma$ une constante.

Il est imagé par le glissement du matériau le long d'une infinité de plans de glissement (shear planes), parallèles aux épontes de la zone et, dans ces plans, suivant une direction constante, celle de glissement (shear direction).

La déformation transforme un cube en un parallélépipède oblique et dans le plan $\mathrm{xz}$, un carré en un parallélogramme (RAMSAY et GRAHAM, 1970). Le glissement $\gamma$ est lié à la distorsion angulaire d'un angle droit dont un côté, initialement parallèle à oz fait (fig. 1), après déformation, un angle $\psi$ avec sa direction initiale, par la relation:

$$
\gamma=\tan \psi
$$

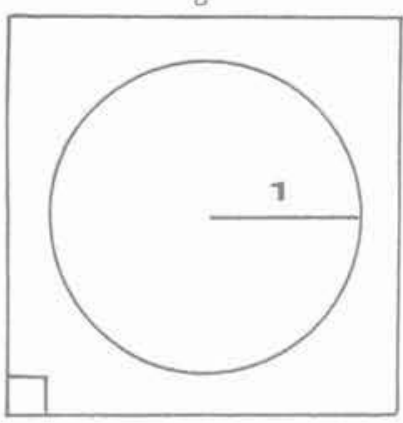

INITIAL $z_{1}$

Fig. 1. - Cisaillement: a. Homogène. b. Hétérogène. Fig. 1. - a. Homogeneous shear. b. Heterogeneous shear.

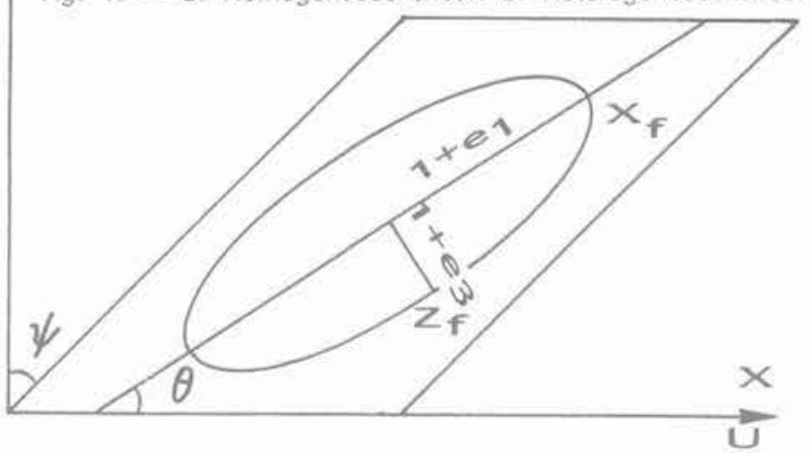

DEFORME

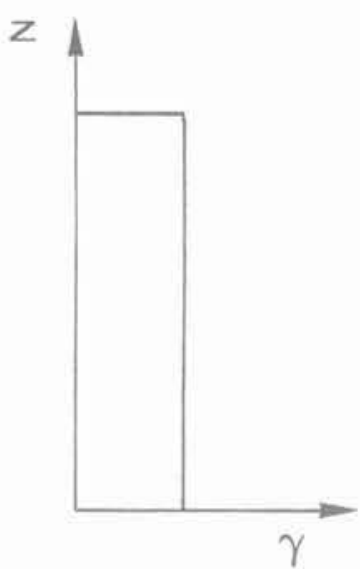

b

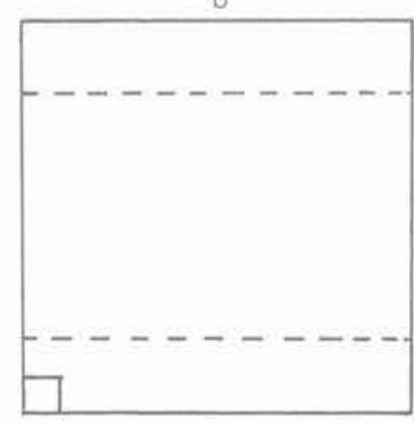

INITIAL

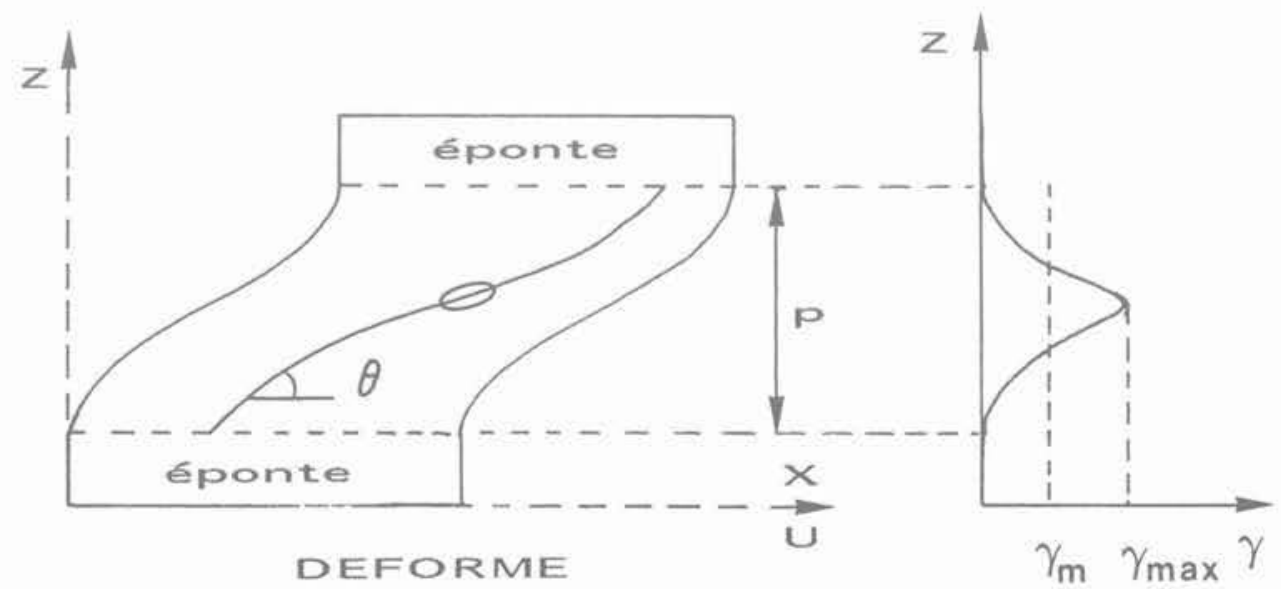


Les déformations principales sont $e_{1}, e_{2}, e_{3}$, (une sphère unitaire contenue dans ce cube est transformée en ellipsoïde de demi-axes $1+e_{1}, 1+e_{2}$, $1+e_{3}$ ) avec :

$$
\begin{aligned}
e_{1} & \geq e_{2} \geq e_{3} \\
\text { et } & \left(1+e_{1}\right)^{2}=\left[2+\gamma^{2}+\gamma\left(\gamma^{2}+4\right)^{1 / 2}\right] / 2 \\
& e_{2}=0 \\
\left(1+e_{3}\right)^{2} & =\left[2+\gamma^{2}-\gamma\left(\gamma^{2}+4\right)^{1 / 2}\right] / 2
\end{aligned}
$$

avec la relation d'invariance de volume

$$
\left(1+e_{1}\right)\left(1+e_{3}\right)=1 \text {. }
$$

Les axes principaux de la déformation incrémentale $X_{i}, Y_{i}, Z_{i}$ sont orientés, selon $y$ et dans $x z$, à $\pi / 4$ et $3 \pi / 4$ de la direction de glissement. Initialement (pour $\gamma$ petit), les axes principaux de la déformation finie $\left(X_{f}, Y_{f}, Z_{f}\right)$ coïncident avec ceux de la déformation incrémentale $\left(X_{1}, Y_{i}, Z\right)$. Mais, dès que la déformation évolue (lorsque $\gamma$ croît), ils ne sont plus en coïncidence, le matériau devenant, en outre, anisotrope.

L'orientation des axes principaux qui correspond aux lignes de déformation principale est donnée par: $\theta=\left(\mathrm{x}, \mathrm{X}_{\mathrm{f}}\right)$ tel que

$$
\tan 2 \theta=2 / \gamma=2 \cot \psi
$$

Dans le cisaillement hétérogène, le champ de déplacement a mêmes expressions, mais $\gamma=\mathrm{du} / \mathrm{dz}$ n'est plus constant mais fonction de $z$ seulement, soit $\gamma(z)$ et nous utiliserons cette formulation. Les relations précédentes restent valables et les quantités $\psi, \theta, e_{1}$ et $e_{3}$ sont alors fonction de $z$.

\section{CARACTÉRISTIQUES DES ROCHES DÉFORMÉES DANS LES ZONES DE CISAILLEMENT}

Les études des zones de cisaillement ont été réalisées dans des roches de nature et de caractères différents mais pouvant être considérées comme initialement isotropes: une granodiorite (fig. 2a), roche siliceuse polyminérale et un calcaire (fig. $2 \mathrm{~b}$ ), roche carbonatée monominérale.

A l'extérieur des zones de cisaillement, la granodiorite du Néouvielle est composée de $30 \%$ de quartz, $55 \%$ de feldspath et $15 \%$ de ferro-magnésiens, les cristaux sensiblement homométriques ayant une dimension moyenne de 1 à $2 \mathrm{~mm}$. Les grains de quartz ont un allongement A (longueur/largeur) de l'ordre de 1 et une extinction régulière ou roulante. La distribution des composants est homogène, sans concentration particulière ni orientation associée à une structure magmatique originelle; à l'échelle des zones de cisaillement la granodiorite représente un matériau homogène et isotrope (fig. 2a).

Les zones de cisaillement apparaissent comme des lentilles de teinte bleutée, très allongées (plusieurs mètres à dizaines de mètres), aux limites tranchées, sensiblement planes et parallèles, de quelques centimètres d'épaisseur. A l'intérieur de la zone, la folia-
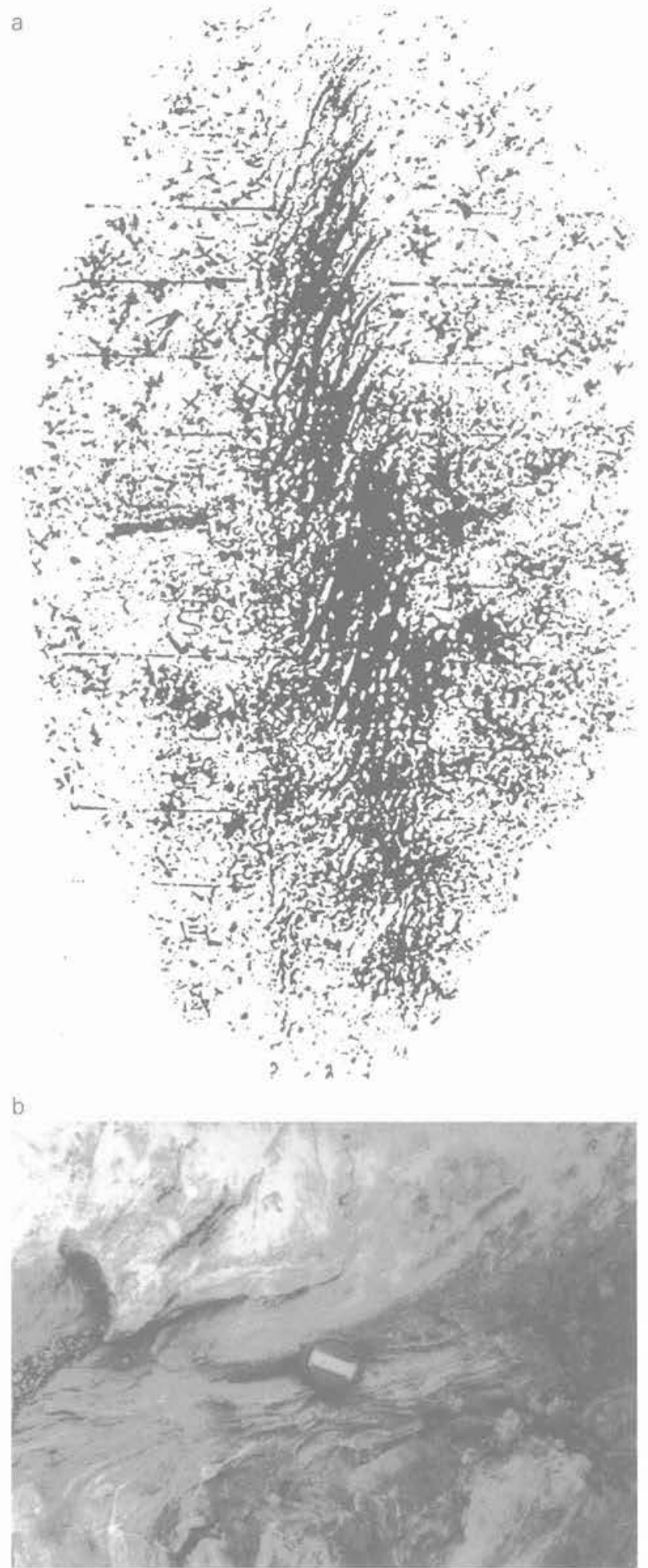

Fig. 2. - Aspect de zones de cisaillement. a. Dans la granodiorite. b. dans le calcaire. Fig. 2. - Aspects of shear zones.

a. In the granodiorite, $b$. In the limestone.

tion globalement sigmoïde (avec donc un point ou une zone d'inflexion) est caractérisée par l'orientation préférentielle des crisaux les plus compétents (feldspaths, cristaux ferro-magnésiens) et l'étirement des cristaux les plus déformables (quartz). A proximité des épon- 
tes, la foliation est fruste et $\theta$ est voisin de 40 à $45^{\circ}$ alors qu'au point d'inflexion l'angle $\theta$ décroît jusqu'à 10-5 ${ }^{\circ}$. L'examen au microscope montre, selon la foliation, une variation continue des caractères cristallins, notamment de la morphologie et de la granulométrie des cristaux de quartz (phénomène déjà observé par SIMPSON, 1983: LAMOUROUX, 1987 ; LAMOUROUX et al., 1991). Près des épontes, la roche est une protomylonite avec des cristaux de quartz de taille moyenne et peu allongés $(A \leq 2)$. Dans la zone d'inflexion, la roche est une orthomylonite avec des cristaux de quartz de petite taille et très allongés $(A>4)$ et des baguettes d'amphibole boudinées.

Le calcaire de la Maladetta est constitué quasi exclusivement de calcite et $a$, hors des zones, un caractère massif et isotrope. Les zones de cisaillement (fig. $2 \mathrm{~b}$ ) ont des épaisseurs décimétriques, la foliation sigmö̈de étant soulignée par l'alternance de lits clairs et sombres, de composition identique.

\section{MESURES DE LA DÉFORMATION (ET DU DÉPLACEMENT)}

La quantification de la déformation et du déplacement, au sein de zones naturelles se fait par l'analyse géométrique des marqueurs de la déformation (RAMSAY et GRAHAM, 1970), induits par le cisaillement (foliation, fentes de tension) ou préexistants (foliations antérieures, filons, surface de contact, ...). Ainsi, il est fréquent d'observer des structures foliées définies par l'orientation morphologique préférentielle de cristaux (antérieurs ou contemporains de la déformation). Ces structures sont assimilées à la foliation et, dans le plan xoz, leurs traces sont alors confondues avec la courbe enveloppe de la direction principale d'extension. Par ailleurs, dans ce même plan, la trace des épontes est considérée comme direction de glissement. Compte tenu de ces deux postulats, il est possible de déterminer, en divers points de la zone, l'angle $\theta$ entre la direction de glissement et celle d'extension finie. Ainsi, le déplacement relatif des épontes correspond d'après (1) à la sommation :

$$
\mathrm{u}=\int_{0}^{\mathrm{p}} \gamma \mathrm{dz}
$$

Cette intégrale, égale à la surface sous-tendue par la courbe $\gamma(\mathrm{z})$ (fig. 1), déterminée par la méthode de SIMPSON (SIBONY et MARDON, 1984) qui consiste à diviser l'épaisseur $\mathrm{p}$ en $(2 n)$ intervalles de largeur constante $\mathrm{m}$, avec $\mathrm{m}=\mathrm{p} / 2 \mathrm{n}$, a pour valeur :

$$
\begin{aligned}
& u=(m / 3)\left[z_{1}+z_{2 n+1}+2\left(z_{3}, \ldots, z_{2 n-1}\right)\right. \\
& \left.+4\left(z_{2}+\ldots+z_{2 n}\right)\right]
\end{aligned}
$$

Le déplacement peut être défini en utilisant d'autres marqueurs tels que fentes de tension, stratification, foliations antérieures, filons, (RAMSAY et GRAHAM, 1970 ; RAMSAY, 1980 ; INGLĖS, 1985 ; WEIJERMAS, 1987) qui n'apparaissent pas dans nos exemples naturels; $\gamma$ et $u$ seront donc obtenus en utilisant les relations (4) et (5).

\section{RÉSULTATS}

L'étude de la déformation au sein de la zone est donc basée sur l'analyse de la variation de l'angle $\theta$ entre la foliation et les épontes. Les mesures ont été effectuées, dans les deux types de roches, dans la portion médiane des zones, c'est-à-dire loin des extrémités, et seuls ont été retenus les exemples où les variations de volume sont négligeables sinon nulles (relativement aux moyens d'analyse utilisés) en accord avec le champ (1).

\subsection{Dans la granodiorite}

Dans une zone d'épaisseur centimétrique, dix trajectoires de foliation régulièrement espacées suivant $\mathrm{x}$, ont été tracées. L'angle $\theta$ a été mesuré aux intersections de ces trajectoires avec une famille de lignes équidistantes parallèles aux épontes, (fig. 3a). Les résultats obtenus sont (fig. $3 \mathrm{~b}$, tab. I) les suivants : - la forme sigmoïde de la foliation se marque par une zone d'inflexion, d'étendue plus ou moins grande, non systématiquement située à mi-distance des épontes, qui se traduit par un palier bien individualisé de la courbe $\gamma(z)$, caractéristique d'une sous-zone à déformation homogène ; la courbe $\gamma(\mathrm{z})$ a une forme assimilable à celle d'un trapèze ;

- les valeurs extrêmales $\gamma_{\max }$ de 4,49 et de 2,65 correspondent respectivement à des extensions $e_{1}$ de $370 \%$ et de $200 \%$;

- Pangle $\theta$ et donc le glissement $\gamma$ et le déplacement $u$, présentent des fluctuations en fonction de $\mathrm{x}$ (fig. $3 b$ et $6 a)$.

\subsection{Dans le calcaire}

La caractérisation précise des trajectoires de foliation étant difficile, l'angle $\theta$ a été mesuré aux nœuds d'un réseau orthogonal constitué par deux familles de lignes équidistantes parallèles et perpendiculaires aux épontes (fig. 4a). Les huit résultats obtenus, dans la zone ayant une puissance de l'ordre de $40 \mathrm{~cm}$ (fig. $4 \mathrm{~b}$, tab. II), montrent que :

- la forme sigmoïde et irrégulière de la foliation montre une zone d'inflexion non située à mi-distance des épontes, d'étendue très réduite, et assimilable à un point; la courbe $\gamma(\mathrm{z})$ présente un pic, et n'a pas de sous-zone à déformation homogène ;

- la variation, avec $z$, de $\gamma$ est donc très hétérogène, les valeurs élevées étant localisées dans une sous-zone aux limites paralleles aux épontes; les valeurs extrêmales $\gamma_{\max }$ de 7,46 et de 3,76 correspondent respectivement à une extension $e_{1}$ de $659 \%$ et de $300 \%$;

- la variation de $\mathrm{u}$ en fonction de $\mathrm{x}$ se traduit par une courbe fluctuante globalement croissante.

Finalement, les mesures effectuées dans des zones de cisaillement affectant des roches différentes mettent en évidence des analogies entre les structures (morphologie générale des zones, caractère sigmoïde de la foliation) mais aussi des différences importantes (étendue de la zone d'inflexion, valeur maximale et hétérogénéité de $\gamma$ ). 

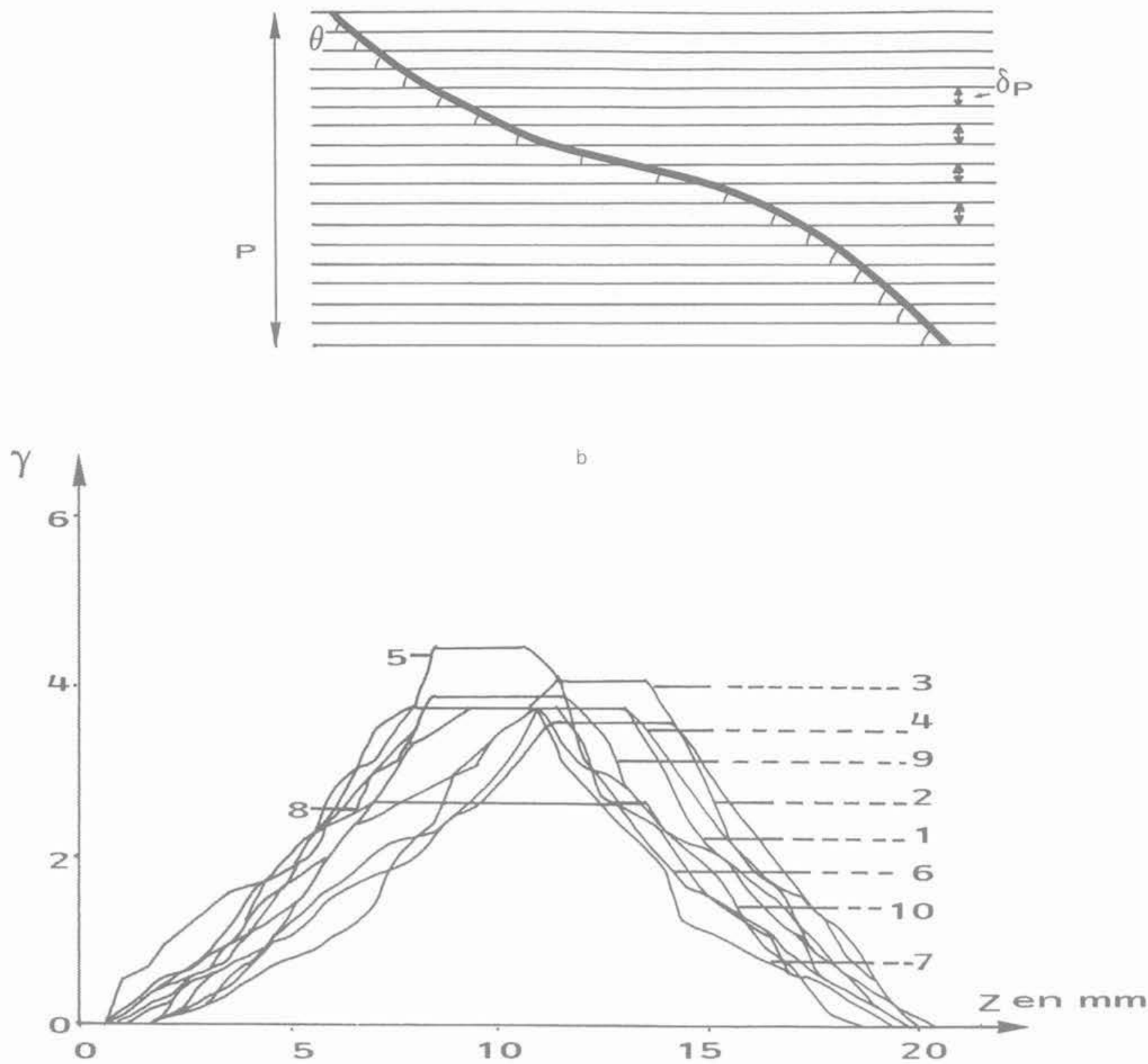

Fig. 3. - Valeurs de y dans la granodiorite: a. Méthode. b. Résultats.

Fig. 3. - Values of $\gamma$ in the granodiorite: a. Method b. Results.

Tableau I. - Résultats des mesures dans la granodiorite.

Table 1. - Results of measures in the granodiorite.

\begin{tabular}{|c|l|l|l|l|l|}
\hline $\begin{array}{c}\mathrm{X} \\
(\mathrm{mm})\end{array}$ & $\theta \mathrm{m}$ & $\begin{array}{c}\mathrm{U} \\
(\mathrm{mm})\end{array}$ & $\gamma \mathrm{m}$ & $\mathrm{h}$ & $\gamma_{\max }$ \\
\hline 10 & 24,7 & 34,4 & 1,72 & 2,19 & 3,76 \\
20 & 24,6 & 34,6 & 1,73 & 2,09 & 3,61 \\
30 & 23,5 & 37,4 & 1,87 & 2,19 & 4,1 \\
40 & 22,9 & 39,0 & 1,95 & 1,93 & 3,76 \\
50 & 21,7 & 42,1 & 2,11 & 2,13 & 4,49 \\
60 & 24,7 & 34,3 & 1,72 & 2,19 & 3,76 \\
70 & 24,0 & 36,0 & 1,8 & 2,09 & 3,76 \\
80 & 25,8 & 31,5 & 1,58 & 1,68 & 2,65 \\
90 & 23,1 & 38,4 & 1,92 & 2,04 & 3,92 \\
100 & 22,9 & 38,7 & 1,94 & 1,94 & 3,76 \\
\hline
\end{tabular}

Tableau II. - Résultats des mesures dans le calcaire. Table II. - Results of measures in the limestone.

\begin{tabular}{|c|c|c|c|c|c|}
\hline $\begin{array}{c}\mathrm{X} \\
(\mathrm{mm})\end{array}$ & $\theta \mathrm{m}$ & $\begin{array}{c}\mathrm{U} \\
(\mathrm{mm})\end{array}$ & $\gamma \mathrm{m}$ & $\mathrm{h}$ & $\gamma_{\max }$ \\
\hline 110 & 34,3 & 344 & 0,78 & 5,26 & 4,10 \\
220 & 32,2 & 421 & 0,96 & 4,47 & 4,29 \\
330 & 28,7 & 564 & 1,28 & 4,29 & 5,49 \\
440 & 29,8 & 516 & 1,17 & 3,21 & 3,76 \\
550 & 28,2 & 585 & 1,33 & 3,38 & 4,49 \\
660 & 25,8 & 696 & 1,58 & 3,90 & 6,16 \\
770 & 22,6 & 874 & 1,99 & 3,75 & 7,46 \\
880 & 27,8 & 595 & 1,35 & 3,66 & 4,95 \\
\hline
\end{tabular}


a

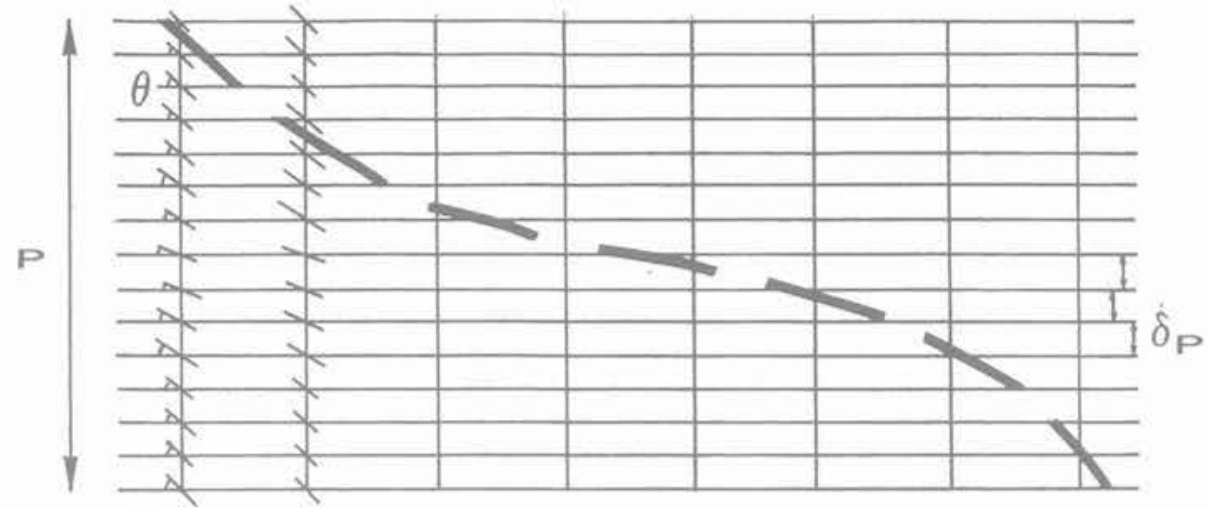

b

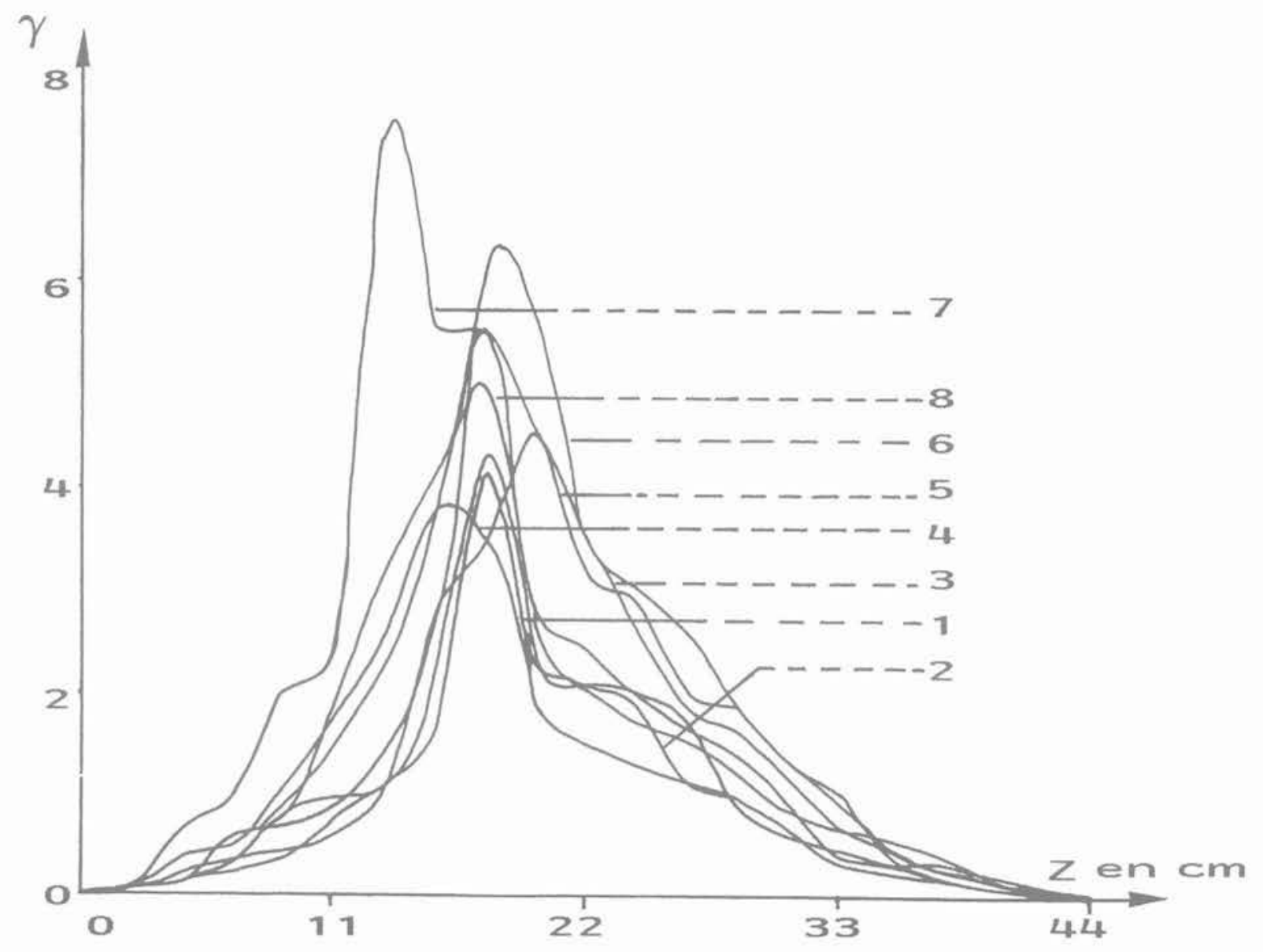

Fig. 4. Valeurs de $\gamma$ dans le calcaire: a. Méthode. b. Résultats.

Fig. 4. - Values of $\gamma$ in the limestone: a. Method. b. Results.

\section{HÉTÉROGÉNÉITÉ DE LA DÉFORMATION}

L'hétérogénéité de la déformaiton dans les zones de cisaillement mérite une analyse particulière.

\subsection{Glissement moyen}

La comparaison des deux zones de cisaillement (d'épaisseurs très différentes 2 et $40 \mathrm{~cm}$ ) s'effectue à 
l'aide d'un premier paramètre : le glissement moyen $\gamma_{\mathrm{m}}$ (celui qui correspond au cas d'un cisaillement homogène où le glissement est constant et la foliation rectiligne) caractérisé par :

$$
\gamma_{\mathrm{m}}=\mathrm{u} / \mathrm{p}
$$

Les résultats, pour les deux types de roches, sont très comparables (tab. I et tab. II). Les valeurs de $\gamma_{m}$ dans la granodiorite sont (à une exception près) comprises dans la fourchette de celles, plus dispersées, du calcaire. En outre, la moyenne des valeurs de $\gamma_{\mathrm{m}}$ pour chaque zone, soit $\gamma_{M}$, est du même ordre de grandeur: 1,31 (calcaire) et 1,83 (granodiorite).

Ainsi, le glissement moyen dans les zones de cisaillement de ces deux types de roches ayant des valeurs voisines, nous pouvons comparer, pour chacune, l'hétérogénéité de glissement, matérialisée par la géométrie des courbes $\gamma(z)$.

\subsection{Facteur d'hétérogénéité}

Dans les deux zones de cisaillement, la déformation moyenne est voisine, mais les courbes $\gamma(\mathrm{z})$ sont différentes. Cette variation est caractérisée par un deuxième paramètre $\mathrm{h}$, le facteur d'hétérogénéité, tel que :

$$
\mathrm{h}=\gamma_{\max } / \gamma_{\mathrm{m}}
$$

Pour la granodiorite (tab. I et tab. II), h varie entre 1,68 et 2,19 (valeur moyenne 2) et pour le calcaire, entre 3,21 et 5,26 (valeur moyenne 4). Cette différence entre les moyennes (de 2 à 4 ) est liée au type de courbe $\gamma(z)$, trapézoïdale ou avec un pic.

Dans la granodiorite, les courbes $\gamma(z)$ ont une forme en trapèze (fig. 5a) caractérisée par deux catégories de sous-zones:

- une sous-zone centrale, qui occupe 15 à $25 \%$ de l'épaisseur $\mathrm{p}$ de la zone, où $\gamma$ est constant, ce qui indique un cisaillement simple (glissement homogène) ;

- deux sous-zones latérales, de part et d'autre de la sous-zone centrale, où le gradient de $\gamma$ suivant $z$ est constant (de lordre $0,5 \mathrm{~mm}^{-1}$ ).

Dans le calcaire, en revanche, les courbes $\gamma(\mathrm{z})$ ont une allure gaussienne avec des sous-zones aux limites plus ou moins bien définies (fig. $5 b$ ) :

- une sous-zone centrale avec un pic élevé et un gradient de $\gamma$ voisin de $0,1 \mathrm{~mm}^{-1}$;

- deux sous-zones latérales avec un faible gradient de $\gamma$ (environ dix fois plus petit que celui de la souszone centrale).

\subsection{Variation longitudinale du glissement moyen}

L'interprétation des mesures de terrain est effectuée à l'aide du champ (1) pour lequel le glissement $\gamma$ (donc le déplacement $\mathrm{u}$ ) est indépendant de $\mathrm{x}$, direction du glissement (et de y). Il convient d'examiner dans quelle mesure cette hypothèse de travail se trouve vérifiée.

Pour la granodiorite (fig. 6a), la valeur moyenne de $u$ est de $36,8 \mathrm{~mm}$ (avec un écart maximal de $\pm 14 \%$ ). Sur la longueur de la zone mesurée, la déformation par glissement s'accompagne d'une défor-
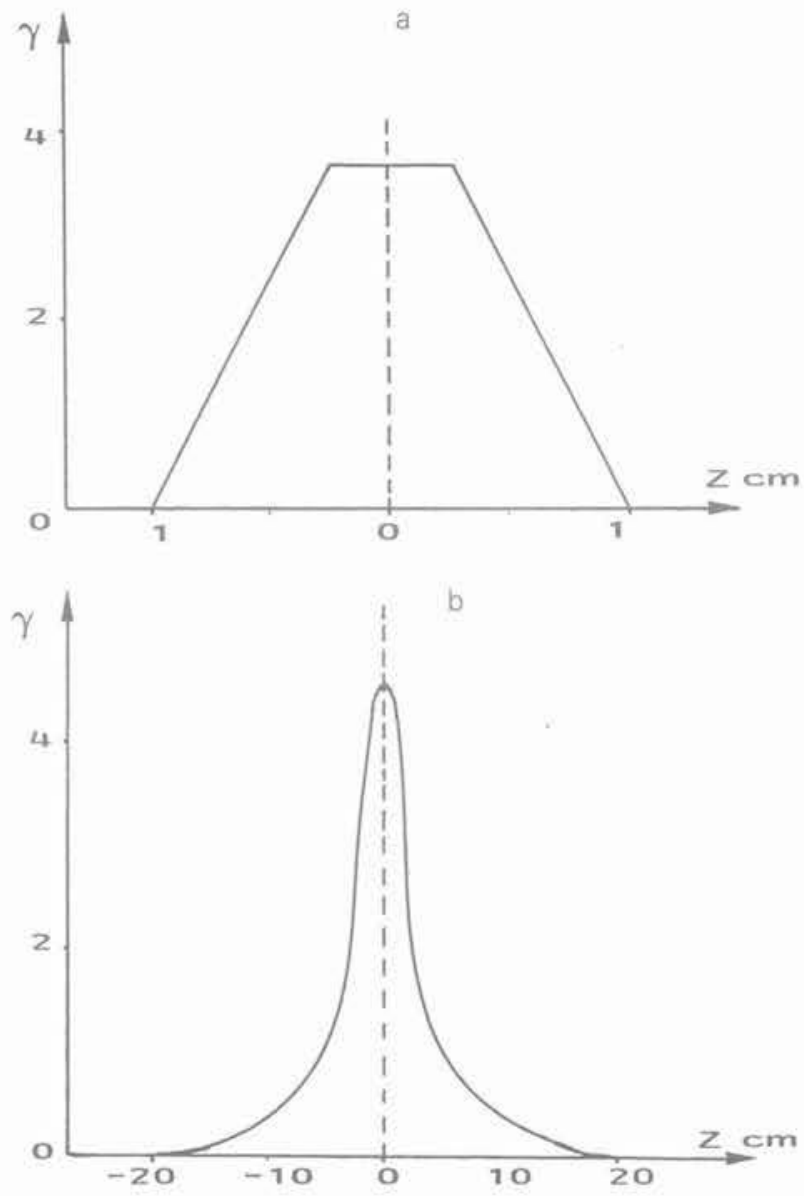

Fig. 5, - Hétérogénéité de la déformation; courbes $\gamma(z)$. a. Trapèze, b. Allure gaussienne,

Fig. 5 . - Deformation heterogeneity : $\gamma(z)$ curves

a. Trapezium, b. Triangular (gaussian shape).

mation moyenne selon Ox égale à $4,7 \%$, évidemment négligeable en regard des valeurs des extensions majeures obtenues par glissement (supérieures à $200 \%$ ) justifiant la relation (1).

Dans le calcaire (fig. $6 \mathrm{~b}$ ), en revanche, $\mathrm{u}$ a des variations plus importantes jusqu'à $43 \%$ autour de la valeur moyenne $60,9 \mathrm{~mm}$ ) ; son gradient (c'est-à-dire la déformation moyenne parallèle à $\mathrm{Ox}$ ) est de $38,6 \%$, valeur certes faible en regard des déformations de cisaillement atteignant $300 \%$. Il conviendrait ici de tenir compte de ce fait dans l'interprétation des résultats, avec une matrice de déformation plus complexe que celle déduite du champ (1). Néanmoins, ces valeurs ne devraient pas modifier de façon significative nos conclusions. Cette roche, plus déformable que la précédente, justifierait de nouvelles mesures.

\section{PROCESSUS DE DÉFORMATION (A L'ÉCHELLE DU GRAIN)}

Dans ces zones de cisaillement et malgré des glissements moyens analogues, l'évolution du glissement est donc très différente, probablement du fait des comportements distincts des deux roches. Ainsi, en première analyse, si le glissement hétérogène résulte de 


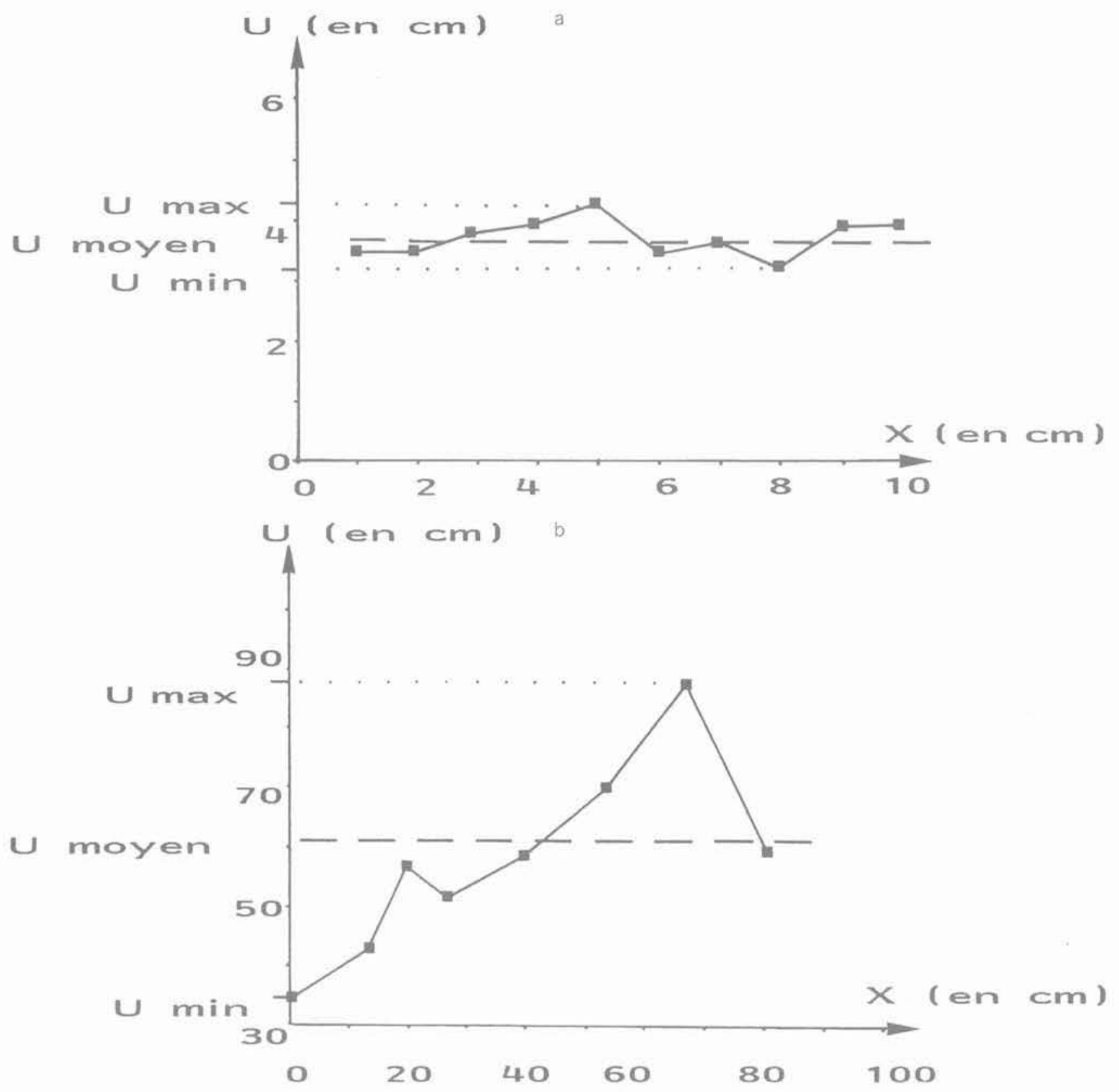

Fig. 6. - Deformation en fonction de x (direction du cisaillement) : a. Granodiorite. b. Calcaire. Fig. 6. - Deformation in the direction of $x$ (shear direction): a. Granodiorite. b. Limestone.

l'activation d'un ensemble de processus de déformation qui affecte les matériaux respectifs, l'apparition de limites-critiques dans le cas de la granodiorite indique l'activation de nouveaux processus spécifiques. Une telle différence pourrait être attribuée, au moins partiellement, à la composition des roches: l'évolution du glissement est hétérogène dans la roche polyminérale (granodiorite) et homogène dans la roche monominérale (calcaire). Cette influence de l'hétérogénéité de la composition minérale de la roche sur l'évolution du glissement et celle de son comportement est basée sur nos résultats et aussi sur ceux d'autres auteurs (RAMSAY et GRAHAM, 1970 ; SIMPSON, 1983b).

Dans la granodiorite, la déformation, dans les souszones latérales, résulte surtout de celle du quartz. A proximité des épontes, elle se marque par la modification de la forme des grains de quartz (SIMPSON, 1983a) et par des mouvements aux limites de tous les cristaux mais surtout des plus compétents (feldspaths). Près des limites de ces sous-zones, l'augmentation de la déformation provoque une importante restauration du quartz marquée par l'apparition de bandes de déformation et une recristallisation localisée suivant la foliation; dans les autres minéraux, moins déformables (feldspaths, biotite, amphibole), se développent des fentes de tension. Dans la sous-zone centrale, la déformation maximale accentue, dans le quartz, la restauration et la recristallisation en fonction de $\gamma_{\max }$ et de la quantité d'eau dont la circulation est facilitée par lanisotropie induite (apparition de la foliation). Dans les autres minéraux, la déforma- 
tion se manifeste par la généralisation du développement de fentes de tension, par des mouvements aux limites de grains et par un début de restauration dans de rares cristaux compétents.

Dans le calcaire, constitué uniquement de grains de calcite, les processus sont identiques dans l'ensemble de la zone :

- mouvements aux limites de grains ; la taille des cristaux, initialement très variée, s'homogénéise vers la sous-zone centrale (c'est-à-dire avec l'accroissement de $\gamma$ ). La multiplication du nombre de grains, en augmentant la longueur des contours de grains de façon quasi-exponentielle, favorise laccentuation de ces mouvements ;

- formation de macles bien développées dans les plus gros cristaux mais également observables dans de petits grains.

Notons qu'au sein d'un métagabbro (constitué de plusieurs minéraux très compétents), RAMSAY et GRAHAM (1970) obtiennent des courbes trapézoïdales lorsque le glissement maximal atteint une valeur critique (de l'ordre de 20). En deçà de cette valeur, la courbe est plutôt du type homogène. Par ailleurs, SIMPSON (1983b) a déterminé, dans des roches granitiques, une évolution homogène du glissement (lhétérogénéité de la compétence des divers minéraux étant très atténuée par les conditions de déformation du faciès amphibolite).

Finalement, c'est plus le constrate de compétence entre les constituants des roches que leur nature qui influe sur l'hétérogénéité de l'évolution du glissement. En conséquence :

- dans les roches composées de minéraux à différence de compétence très marquée, l'accroissement du glissement se marque par une augmentation progressive de la déformation dans les sous-zones latérales où elle affecte des minéraux de plus en plus compétents et qui présentent une ductilité croissante. Pour une valeur critique, $\gamma_{\max }$, la recristallisation du quartz tend à se développer et. le glissement à devenir plus homogène, les mouvements des grains compétents au sein de la matrice constituée par la multitude de petits cristaux étant facilités ;

- dans les roches monominérales (ou constituées de minéraux de compétence voisine), la déformation provoque toujours les mêmes processus.

\section{CONCLUSION}

Au sein de deux types de roches (granodiorite et calcaire), les zones ductiles de cisaillement sont caractérisées par la localisation de la déformation dans des zones peu épaisses (relativement à leur longueur), limitées par des épontes globalement planes. Les traces des surfaces de foliation induites ont une forme sigmoïde dont l'analyse géométrique permet de quantifier la déformation et le déplacement relatif des deux compartiments. Les résultats montrent plusieurs différences, la plus importante étant dans la forme des courbes de glissement, qui ont respectivement une allure trapézoïdale et une allure gaussienne. Cette différence peut être attribuée à l'hétérogénéité de compétence des éléments constitutifs des roches qui dépend, à la fois, de la nature des minéraux et de leur comportement mécanique lui-même fonction notamment des conditions physiques de déformation.

\section{BIBLIOGRAPHIE}

INGLĖS J. (1985), Theoretical and natural strain patterns in ductile simple shear zones. Tectonophysics, Amsterdam, The Netherland, vol. 115, 315-334

LAMOUROUX C. (1976), Les mylonites dans le massif du Néouvielle. Déformations pyrénéennes d'un complexe plutonique hercynien. Thèse présentée à YUniversité Paul-Sabatier (Toulouse), soutenue le 16 octobre 1976 pour obtenir le grade de docteur de $3^{\mathrm{e}}$ cycle, $148 \mathrm{p}$.

LAMOUROUX C. (1987), Les mylonites des Pyrénées. Classification, mode de formation et évo. lution. Thèse présentée à l'Université Paul-Sabatier (Toulouse), soutenue le 6 février 1987 pour obtenir le grade de docteur ès-Sciences, 395 p. Mémoire Société Géologique du Nord, Lille, n० 19, 1991.

LAMOUROUX C., INGLÈS J., DEBAT P. (1991), Conjugate shear zone.Tectonophysics, Amsterdam, The Netherland, vol. 185 , 309-323.

RAMSAY J.G. (1980), Shear geometry : a review. Journal of Structural Geology, Oxford, England, 2, 83-99.

RAMSAY J.G. (1982), Chap. 1.9 Rock ductility and its influence on the development of tectonic struc. tures in mountain belts. In Hsü K.J., Editor, Mountain building processes, Academic Press, London, pages 111-127.

RAMSAY J.G., GRAHAM R.H. (1970), Strain variation in shear belts. Canadian Journal of Earth Sciences, Canada, 7, 786-813.

RAMSAY J.G., ALLISON I. (1979), Structural analysis of shear zones in an alpinised Hercynian gra. nite, Maggia Lappen, Pennine zone, Central Alps. Schweizer mineralogisches petrographisches Mittelungen, Suisse, 59, 251-279.

RAMSAY J.G., HUBER M.I. (1987), Session 26 Ductile and Brittle Shear Zones. In Ramsay J.G. \& Huber M.I., Editors, The techniques of modern structural geology, vol. 2, Academic Press, London, pages 595-640.

SIBONY M., MARDON J.C. (1984), Ch. IV: Approximation, interpolation, dérivation et intégration numérique. In Approximations et équations différentielles. Edition Hermann, Paris, pages IV.2-IV. 185

SIMPSON C. (1983a), Strain and shape fabric variations associated with ductile shear zones. Journal of Structural Geology, Oxford, England, 5, 61-72.

SIMPSON C. (1983b), Displacement and strain patterns from naturally occuring shear zone terminations. Journal of Structural Geology, Oxford, England, 5, 497-506.

WEIJERMARS R. (1987), The construction of shear strain profiles accross brittle-ductile shears. Preliminary estimates of conventional shear strain rates for Truchas and Palomares Shears (Spain) and the Alpine Fault (New Zealand). Annales Geophysicae, EGS-Gauthier-Villars, Paris, 5B, (2), 201-210. 